\title{
Risk factors for readmission to hospital for asthma in childhood
}

\author{
E A Mitchell, J M Bland, J M D Thompson
}

\begin{abstract}
Background - Readmissions account for part of the increase in hospital admissions for asthma in childhood seen in many countries.

Methods - This observational study recorded demographic features and the severity, treatment, and management of asthma in 1034 individual children admitted to hospital over a one year period, followed for a maximum of 33 months. Results - Readmissions were common, with $33 \%$ readmitted by six months and $51 \%$ by two years. After controlling for a wide range of variables, factors that significantly increased readmission were: female sex (relative risk (RR) $1 \cdot 23$; $95 \%$ confidence interval (CI) 1.03 to 1.46 ), young age (age $<5$ years RR $1.71 ; 95 \%$ CI $1 \cdot 41$ to $2 \cdot 08$ ), number of previous admissions (one previous admission $R R$ 1.32; two, RR 1.68; three, RR 2.00; four or more, $R R$ 2.80), and inpatient intravenous treatment (RR 1.29; $95 \%$ CI 1.08 to 1.55). Inpatient treatment with theophylline was used frequently $(98 \cdot 4 \%)$, but was associated with decreased readmissions (RR 0.51, 95\% CI 0.28 to 0.92). Factors which did not predict readmission included ethnicity, respiratory and pulse rate, medical team, prescribed prophylactic treatment, type of follow up, or the use of action plans.
\end{abstract}

Conclusions - Risk factors for readmission relate to the characteristics of the individual (age and sex), severity of the condition (intravenous treatment), and number of previous admissions which may reflect severity or behaviour of the illness. Medical treatment and management did not influence readmissions. Strategies to reduce the high readmission rate for asthma in childhood need to be developed.

(Thorax 1994;49:33-36)

Admissions to hospital for childhood asthma have increased dramatically in many countries over the last two decades. ${ }^{1}$ Although much of this increase is a result of the increase in the number of individual children admitted to hospital, ${ }^{23}$ an increase in the number of readmissions per child accounts for some of this increase in New Zealand. ${ }^{45}$

Case note review of admissions for asthma have described the characteristics of these children. ${ }^{267}$ Many have been admitted previously and some have had many admissions. This study examines the risk factors for readmission of children to hospital for asthma.

\section{Methods}

At the time of this study all children in the Auckland region (total population 900000 ) who required admission to hospital for asthma (ICD 473) were admitted to the children's wards of Auckland Hospital, a teaching hospital with four paediatric medical teams, two with consultants with academic appointments and two without. Children who had previously been in hospital were admitted to the team which had previously cared for them; those being admitted for the first time were cared for by the admitting team of the day.

STUDY DESIGN

The list of discharges for Auckland Hospital by diagnosis for each year was examined. Only those admissions which were principally due to asthma were considered (diagnostic category A). If a child had had more than one admission in the 12 month period from 1 March 1986 to 28 February 1987 the first admission was selected; thus each observation in the data set represents an individual child aged $0-14$ years admitted to Auckland Hospital for asthma.

The medical records were examined and the following information extracted:

1. Demographic variables: sex, race (Maori, Pacific Islander, European and other), age (calculated from date of birth and date of admission).

2. Severity variables: number of previous admissions for asthma excluding the index submission, duration of wheeze before admission (in hours), respiratory rate, pulse rate, and resident staff assessment of severity (mild, mild to moderate, moderate, moderate to severe, severe and admission to intensive care). 3. Variables related to the inpatient treatment of the index episode: clinical team to which admitted, nebulised treatment, intravenous treatment, steroids, theophylline and antibiotics.

4. Variables related to discharge: prescribed long term $\beta$ agonists, long term theophylline and prophylactic therapy (oral steroids or inhaled cromoglycate or inhaled steroids), follow up arrangements (nil, general practitioner, registrar, paediatrician, asthma clinic), and whether or not there was a copy of a written action plan in the records. 


\section{READMISSION}

In December 1988 the computerised medical information system was examined for each patient admitted during the study period. From the computer records the date of any readmission for each subject was ascertained. In addition, for any subject with missing medical records the age, race, address, length of hospital stay, and readmission data were extracted. Thus follow up ranged from 642 days for patients admitted on 28 February 1987 to a maximum of 1006 days for patients admitted on 1 March 1986 . If the child was readmitted the date of the first readmission after the index admission was recorded. From this was calculated the length of time (in days) to readmission from the date of discharge from hospital of the index admission. Those not readmitted were censored at the date of the last readmission, 19 November 1988.

\section{DATA ANALYSIS}

The data were analysed by Cox regression ${ }^{8}$ using the statistical program STATA (Computing Resource Center, Los Angeles, California, USA). The number of previous admissions is very highly skewed with $40 \%$ of the subjects not having been admitted previously with asthma. Its distribution is not transformable to a normal distribution, but a square root transformation was used to reduce the skewness.

For calculation of admission rates the 1986 census data for the Auckland region, which

Table 1 Distribution of variables (mean, median and range, or number and percentage as appropriate) in 1034 children

\begin{tabular}{lc}
\hline Demographic & \\
Sex & \\
Male & $619(59 \cdot 9 \%)$ \\
Female & $415(40 \cdot 1 \%)$ \\
Race & \\
European & $477(46 \cdot 3 \%)$ \\
Maori & $263(25 \cdot 5 \%)$ \\
Pacific Islander & $264(25 \cdot 6 \%)$ \\
Other & $27(2 \cdot 6 \%)$ \\
Age (years) & $4 \cdot 5,3 \cdot 4,0 \cdot 3-11 \cdot 4$ \\
Severity of asthma & \\
Number of previous admissions & $2 \cdot 7,0,0-83$ \\
Duration of wheeze before admission (hours) & $37 \cdot 3,24,0-720$ \\
Respiratory rate (breaths/min) & $44 \cdot 3,42,16-96$ \\
Pulse rate (beats/min) & $136,140,78-204$ \\
Severity assessment (of 875 children) & $220(25 \cdot 1 \%)$ \\
Mild & $140(16 \cdot 0 \%)$ \\
Mild to moderate & $307(35 \cdot 1 \%)$ \\
Moderate & $157(17 \cdot 9 \%)$ \\
Moderate to severe & $39(4 \cdot 5 \%)$ \\
Severe & $12(1 \cdot 4 \%)$ \\
Intensive care & \\
Treatment of index admission & \\
Clinical team & \\
Medical team 1 (academic) & \\
Medical team 2 & $223(21 \cdot 9 \%)$ \\
Medical team 3 & $265(26 \cdot 1 \%)$ \\
Medical team 4 (academic/respiratory) & $280(27 \cdot 5 \%)$ \\
Nebulised $\beta$ agonist & $249(24 \cdot 5 \%)$ \\
Intravenous therapy & $1024(99 \cdot 0 \%)$ \\
Steroid & $584(56 \cdot 6 \%)$ \\
Theophylline & $655(63 \cdot 4 \%)$ \\
Antibiotics & $1017(98 \cdot 4 \%)$ \\
Discharge from hospital & $271(26 \cdot 3 \%)$ \\
Regular $\beta$ agonists & \\
Regular theophylline & \\
Prophylactic therapy & $855(82 \cdot 7 \%)$ \\
Follow up arrangements & $655(63 \cdot 3 \%)$ \\
Nil & $386(37 \cdot 3 \%)$ \\
General practitioner & $100(9 \cdot 7 \%)$ \\
Registrar & $671(65 \cdot 0 \%)$ \\
Respiratiary paediatrician (asthma clinic) & $69(6 \cdot 7 \%)$ \\
Copy of action plan in medical records & $140(13.6 \%)$ \\
\hline & $52(5 \cdot 0 \%)$ \\
& $157(15 \cdot 2 \%)$ \\
\hline
\end{tabular}

coincides with Auckland Hospital's catchment area for children, was used as the denominator.

\section{Results}

In the 12 months from 1 March 1986 to 28 February 19871034 children aged 0-14 years were admitted to the children's wards at Auckland Hospital for asthma. Due to readmissions of the same patient the total number of admissions for asthma in the 12 month period was 1347 , giving an age specific admission rate of $654 / 100000$. There were no deaths. The average percentage of missing data per variable was $1 \cdot 4 \%$.

The distribution of the demographic data, severity of asthma, and inpatient treatment, and the variables related to discharge from hospital in the 1034 children admitted with asthma, are shown in table 1.

The proportion of children readmitted for asthma over the study period are shown in the figure. At three months $23 \%$ of children had been readmitted, at six months $33 \%$, at one year $43 \%$, and at two years $51 \%$.

The first analysis using Cox regression included the demographic variables of sex, race, and age. All were related to readmission. The risk was increased significantly by being female, a Maori or Pacific Islander, and young. All these variables were retained in subsequent analyses.

The second analysis, in addition, included the following severity variables: square root of the number of previous admissions, duration of wheeze before admission, respiratory and pulse rate, and severity assessment by the resident medical staff. Of these, the square root of number of previous admissions and severity assessment were significantly related to the increased risk of readmission and were, therefore, retained in subsequent analyses.

The third analysis also included the inpatient treatment variables, clinical team, nebulised treatment, steroids, theophylline, and antibiotics. Intravenous therapy was associated with a significantly increased risk of readmission and theophylline with a decreased risk, so both were retained in the subsequent analysis. In the analysis the severity assessment variable is no longer significant, as severity assessment and intravenous treatment are closely related. As there were 159 missing values $(15.4 \%)$ for severity assessment it was dropped from the model.

The next analysis included variables related to discharge from hospital, namely long term use of $\beta$ agonists, long term treatment with theophylline, prophylactic therapy, follow up arrangements, and action plans. None of these variables were related to readmission. After adjustment for sex, age, previous admissions, intravenous treatment, and inpatient treatment with theophylline, action plans were not related to admission (RR $1 \cdot 19 ; 95 \%$ CI 0.94 to $1 \cdot 50)$.

Table 2 shows the variables used in the final analysis. Relative risks for readmission are shown unadjusted and adjusted for the other 
Table 2 Variables independently related to readmission to hospital for asthma in childhood (odds ratios and $95 \%$ confidence intervals)

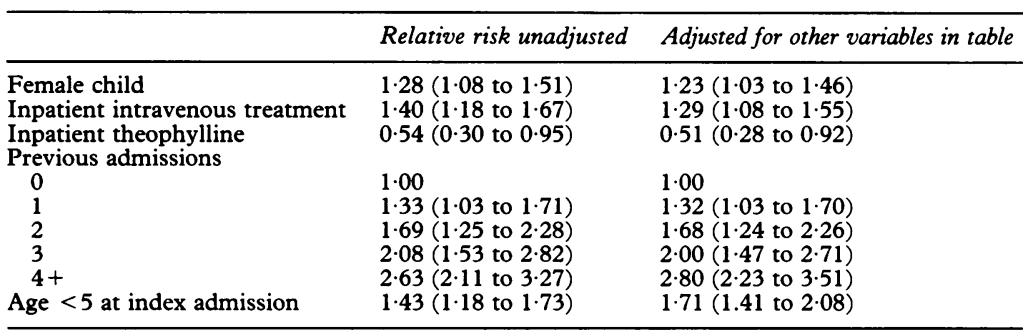

variables in the final model. The risk of readmission was increased by female gender, young age, inpatient intravenous treatment, and number of previous admissions, but decreased by inpatient treatment with theophylline. The number of previous admissions has been grouped to provide more meaningful relative risks. The main effect of adjustment was to increase the effects of age and of previous admissions. This was because the two variables were correlated $(r=0.31)$. The best single predictor of readmission was number of previous admissions. This is illustrated graphically in the figure.

\section{Discussion}

Asthma is the most common medical cause for admission to hospital in childhood. Furthermore, readmission to hospital for asthma is very frequent. In this study we found that only $40 \%$ of children admitted to hospital for asthma have not been admitted previously for asthma. Following the index admission $33 \%$ of children will be readmitted by six months, rising to $51 \%$ at two years. Examination of the figure also suggests that a plateau has not been reached, so that if the children were followed for longer more would have been readmitted. These readmission rates, although high, are of the same magnitude as those reported from three centres in the United Kingdom. ${ }^{269}$

Risk factors for admission to hospital for asthma in New Zealand have previously been reported. ${ }^{4}$ Maoris and Pacific Islanders both have an increased risk of admission compared

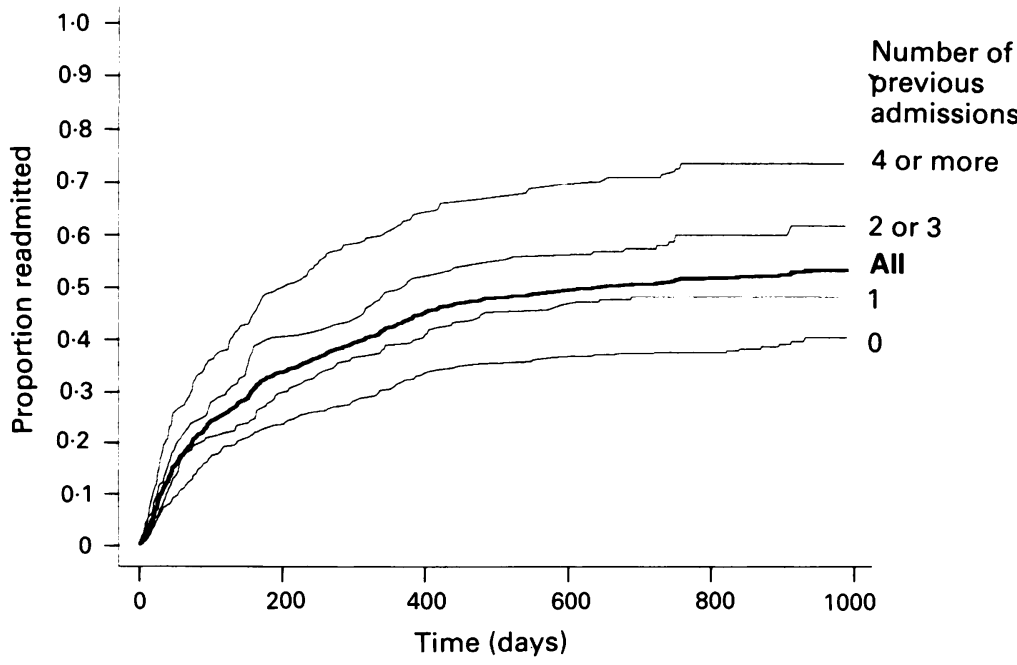

Proportion of subjects readmitted $v$ time (Kaplan-Meier survival curves) according to number of previous admissions. with Europeans. Similarly, the younger child has an increased risk of admission compared with older children, and boys have an increased risk of admission for asthma compared with girls. Similar results have been seen in other countries.

Limitations in this study should be recognised. Factors other than severity of illness influence the decision to admit or readmit. These include time of day, distance from hospital, and family psychosocial factors. These factors were not studied. Medical management of the asthma episode in the community could not be assessed as drug treatment before admission to hospital was poorly recorded. Furthermore, we have no information as to whether prophylactic drugs were taken, clinics attended, or action plans followed. However, what we are assessing here is the "intention to treat." Young children have an increased risk of readmission compared with older children. This finding is perhaps not surprising as management of preschool children is difficult and preventive therapy may be unhelpful. In contrast to admissions, girls have a greater risk of subsequent readmissions than boys. This unexpected finding is unexplained.

The risk of readmission is increased by the severity of the index admission (the doctor's subjective assessment of severity and intravenous therapy). Although assessment of severity may be difficult, especially for relatively inexperienced junior doctors, these subjective assessments were made prospectively. However, we were unable to show that respiratory or pulse rate provided any additional information. It should, however, be borne in mind that these variables are closely related and doctors may have used respiratory and pulse rate as part of their assessment of severity.

The number of previous admissions for asthma was found to be associated with a significantly increased risk of readmission. It has previously been shown that an admission in the previous 12 months is a strong marker of subsequent risk of readmission and death. ${ }^{10}$ As well as a measure of severity, this variable may be a measure of the parent's ability to assess and utilise health care.

The treatment of the index admission had little bearing on the risk of readmission apart from the use of intravenous treatment and the use of theophylline. Intravenous treatment is probably a marker of severity. Theophylline was used in most patients $(98.4 \%)$ and was unexpectedly associated with a decreased risk of readmission. This finding was unexpected but, if true, it has potentially important consequences as the use of theophylline in the acute management of asthma has decreased in Auckland and in other centres over subsequent years. ${ }^{11-13}$ Other factors, including the medical team to which the child was admitted, had no influence on the subsequent outcome.

The prescribed drugs used in the management of the asthma somewhat surprisingly had little effect on the chance of readmission. In particular, one might have expected that inhaled steroids or cromoglycate would have 
reduced the likelihood of readmission, but this was not seen. This was not, however, a randomised controlled study and use of prophylactic treatment may be related to perceived severity and number of previous admissions. It should also be noted that we did not measure compliance with the prescribed treatment. Concern has recently been expressed about the possible adverse effects of regular use of $\beta$ agonists, ${ }^{14}$ but we were unable to show an associated increased risk of readmission with these drugs.

The follow up arrangements of the patients had no influence on outcome. This was unexpected as one might have postulated that the asthma clinics which have been established by many paediatric and medical services and have recently been recommended in the United Kingdom might provide optimal care and thus reduce the likelihood of readmission. We would recommend that asthma clinics are formally evaluated in controlled studies.

Finally we addressed the issue of action plans which have been promoted as an essential component of asthma management. ${ }^{11215}$ Although the index admissions were in 198687 , action plans were firmly established in Auckland for paediatric asthma patients at that time, with $15 \%$ of subjects having a copy of the individualised action plan in the medical records. It seems likely that more were used but a copy was not attached to the medical records. Their use was closely associated with number of previous admissions and the doctor's assessment of severity of the index admission. After controlling for these factors we were unable to show that they reduced readmissions; in fact, if anything, they slightly increased the risk of readmission (RR $1 \cdot 19 ; 95 \%$ CI 0.94 to 1.50 ). It would not be surprising if action plans did increase readmission rates as most action plans, and certainly those in use in Auckland at this time, instructed the patient or their parents to call an ambulance or to seek urgent medical attention if the relief from their bronchodilator was short lived, or they had difficulty with speaking or were cyanosed. Recent studies have highlighted the prevalence of severe asthma in the community, such as "the child cannot talk in sentences", which does not result in parents seeking medical attention for their child. ${ }^{16}$ Thus these instructions may be shifting medical care from the community to the hospital. The unexpected effects of increasing readmissions in those families being followed by asthma nurse educators has been seen in a controlled study, ${ }^{17}$ although other studies have shown a reduction. ${ }^{18}$ We recommend that action plans are formally evaluated.

In summary, risk factors for readmission of children with asthma relate to the demographic characteristics of the individual (age and sex), severity of the asthma (intravenous treatment), and number of previous admissions which may reflect severity or illness behaviour. Medical treatment and management did not influence readmissions. Strategies to reduce the high readmission rate for asthma in childhood need to be developed.

1 Mitchell EA. International trends in hospital admission rates for asthma. Arch Dis Child 1985;60:376-8.

2 Anderson HR. Increase in hospital admissions for childhood asthma: trends in referral, severity, and readmissions from 1970 to 1985 in a health region of the United Kingdom. Thorax 1989;44:614-9.

3 Vollmer WM, Buist AS, Osborne ML. Twenty year trends in hospital discharges for asthma among members of a health maintenance organisation. $f$ Clin Epidemiol 1992;45:999-1006.

4 Mitchell EA, Cutler DR. Paediatric admissions to Auckland Hospital for asthma from 1970-1980. NZ Med $f$ 1984;97:67-70.

5 Harwood LJ, Dawson KP, Mogridge N. Admission patterns for childhood acute asthma: Christchurch 1974-89. terns for childhood acute as

6 Conway SP, Littlewood JM. Admission to hospital with asthma. Arch Dis Child 1985;60:636-9.

7 Mitchell EA, Burr D. Comparison of the characteristics of children with multiple admission to hospital for asthma with those with a single admission. NZ Med 1987;100:736-8.

8 Cox DR. Regression models and life tables. $\mathcal{f} R$ Stat Soc 1972;34:187-200.

9 Storr J, Barrell E, Lenney W. Rising asthma admissions and self referral. Arch Dis Child 1988;63:774-9.

10 Crane J, Pearce N, Burgess C, Woodman K, Robson B, Beasley R. Markers of risk of asthma death or readmission in the 12 months following a hospital admission for asthma. Int $\mathcal{F}$ Epidemiol 1992;21:737-44.

11 Warner JO, Gotz M, Landau LI, Levison H, Milner AD, Pedersen S, et al. Management of asthma: a consensus statement. Arch Dis Child 1989;64:1065-79.

12 Warner JO, Neijens HJ, Landau LI, Jones K, Ashe MS Rachelefsky GS, et al. Asthma: a followup statement from an international paediatric asthma consensus group. Arch Dis Child 1992;67:240-8.

13 Mitchell EA and Ad Hoc Paediatric Group. Consensus on acute asthma management in children. NZ Med $f$ 1992;105:353-5.

14 Sears MR, Taylor DR, Print CG, Lake DC, Li Q, Flannery EM, et al. Regular inhaled beta-agonist treatment in bronchial asthma. Lancet 1990;336:1391-6.

15 Rachelefsky GS. Review of asthma self-management programme. f Allergy Clin Immunol 1987;80:506-11.

16 Robertson CF, Heycock E, Bishop J, Nolan T, Olinsky A Phelan PD. Prevalence of asthma in Melbourne school children: changes over 26 years. BMF 1991;302:1116-8.

17 Mitchell EA, Ferguson V, Norwood M. Asthma education by community child health nurses. Arch Dis Child 1986;61:1184-9.

18 Mayo PH, Richman J, Harris HW. Results of a program to reduce admissions for adult asthma. Ann Intern Med $1990 ; 112: 864-71$ 